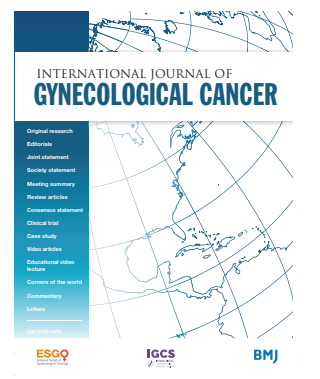

\title{
Intracardiac intravenous leiomyomatosis: diagnosis and management
}

\author{
Ivan Stilidi \\ Director of N.N. Blokhin National Medical Research Center of Oncology, N.N. Blokhin National \\ Medical Research Center of Oncology of the Ministry of Health of the Russian Federation, Moscow, \\ Russian Federation \\ Julia Paianidi \\ Gynecologic Oncology, N.N. Blokhin National Medical Research Center of Oncology of the Ministry of \\ Health of the Russian Federation, Moscow, Russian Federation \\ Vagan Bokhian \\ Department of Tumor Diagnostics, N.N. Blokhin National Medical Research Center of Oncology of the \\ Ministry of Health of the Russian Federation, Moscow, Russian Federation \\ Julia Andreeva \\ Department of Pathology, Russian Medical Academy of Continuing Professional Education, Moscow, \\ Russian Federation \\ Alexey Shevchuk \\ Gynecologic Oncology, N.N. Blokhin National Medical Research Center of Oncology of the Ministry of \\ Health of the Russian Federation, Moscow, Russian Federation \\ Pedro T Ramirez \\ Gynecologic Oncology, MD Anderson Cancer Center, Houston, Texas, USA
}

\begin{abstract}
Correspondence to Dr Alexey Shevchuk, Gynecologic Oncology, N.N. Blokhin National Medical Research Center of Oncology of the Ministry of Health of the Russian Federation, Moscow, Russian Federation; oncogyn@ live.ru
\end{abstract}

Accepted 5 June 2020 Published Online First 7 July 2020

\section{Check for updates}

(C) IGCS and ESGO 2020. № commercial re-use. See rights and permissions. Published by BMJ.

To cite: Stilidi I, Paianidi J, Bokhian V, et al. Int J Gynecol Cancer 2020;30:1243-1247.

\section{CASE PRESENTATION}

A 37-year-old patient presented with recurring episodes of weakness and dizziness, including loss of consciousness, for over 2 years prior to presentation. She denied any gynecologic symptoms and reported a normal menstrual cycle. She denied any medical history. Her past surgical history was significant for myomectomy performed 2 years prior to presentation for a leiomyoma measuring $6 \mathrm{~cm}$ in diameter. At the time of presentation, the physical examination detected a large uterine tumor that occupied the entire pelvic cavity and the greater part of the abdominal cavity.

Discussants: Please provide details as to what should be the initial evaluation of a patient with this presentation

The initial evaluation in this patient should include a computed tomographic (CT) scan of the chest, abdomen, and pelvis, given the enlarged pelvic mass, to evaluate the primary tumor site and to determine if there is evidence of metastatic disease. These imaging modalities are used in order to characterize the complex pelvic mass and to evaluate the soft tissue composition which may provide useful information regarding sites of attachment of the intravenous component. Alternatively, magnetic resonance imaging (MRI) may also be considered; however, motion artifact may be the main disadvantage of
MRI. In the setting of intravenous leiomyomatosis, MRI usually shows a mass of isosignal intensity on T1-weighted imaging and a heterogeneous and highintensity signal on T2-weighted images. ${ }^{1}$ Once there is suspicion of intravenous leiomyomatosis, one should seek additional testing including echocardiography. Trans-esophageal echocardiography has been used to show the details of the inferior vena cava, interatrial septum, tricuspid valve structures, and attachment of the tumor. ${ }^{2}$ Cardiac catheterization prior to surgery is usually not required.

\section{Dr Bokhian}

In the process of surgical planning, a comprehensive imaging examination was performed. CT angiography showed a tumor in the pelvic cavity and tumor thrombus (Figure 1A,B). A massive multinodular uterine tumor $35 \mathrm{~cm}$ in size occupied almost the entire abdominal and pelvic cavity; extension into the retroperitoneal space was also suspected, with inferior vena cava involvement at the level of the renal veins with intravenous growth (tumor thrombus) extending upward by $16 \mathrm{~cm}$. The proximal part of the thrombus was $4.5 \mathrm{~cm}$ in diameter and occupied the largest portion of the right atrium. The uterine tumor received blood supply predominantly from the branches of the uterine, ovarian, right lumbar arteries and capsular arteries of the right kidney. Unlike the atrial myxoma, this tumor has a gross extracardiac component; there 


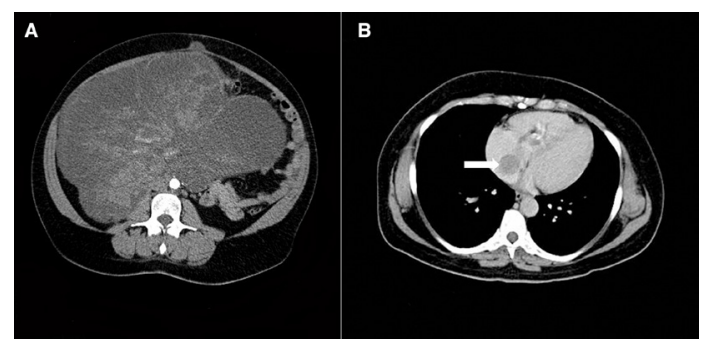

Figure 1 (A, B) CT angiography. Tumor in pelvic cavity and tumor thrombus.

were no tumors in the kidney which allowed for exclusion of a renal cell carcinoma, and attenuation in contrast-enhanced CT allowed us to differentiate it from a routine caval and atrial thrombosis.

Dr Stilidi: What were the details of the discussion at the time of presentation of this patient as to whether to proceed with surgery versus non-surgical management?

Prior to surgery the patient was counseled regarding the extent of the procedure, in particular the necessity to remove the uterus with adnexa, and thus the fact that she would not be able to preserve her fertility. The patient was informed that the prognosis of the disease depended on the radicality of the surgery, including possible resection of all tissues involved in the abdominal and thoracic cavity, and retroperitoneal space. We also discussed with the patient the high probability of using donated blood as a result of potential massive blood loss and multiple blood transfusions.

Discussants: Please provide details as to when to perform the procedure as part of a one-stage versus two-stage approach

The decision to stage the procedures should be based on a number of important factors that include the patient performance status, the physiologic reserve, complexity of the procedure, and the resources available in the hospital. Patients with a diagnosis of intravenous leiomyomatosis should be evaluated and treated by a multidisciplinary team including gynecologic oncologists, anesthesiologists, an intensive care specialist, and thoracic surgeons. The one-stage procedure is generally considered when complete resection of the tumor is feasible through a single extended incision that includes laparotomy and sternotomy. In the first step, tumor resection in the pelvis is performed, along with hysterectomy and adnexectomy and dissection of the inferior vena cava. The second step of the one-stage procedure is to remove the intravenous leiomyomatosis via sternolaparotomy with cardiopulmonary bypass or deep hypothermic arrest. The one-stage procedure has several advantages, including avoidance of hemodynamic complications during the interval between the two surgeries, avoiding the risk of a second general anesthetic, and avoiding the risk of tumor embolism caused by incomplete resection in the first stage.

The two-stage procedure involves removal of the intracardiac tumor first, followed by staged removal of the remaining intraabdominal tumor at a later time. The two-stage procedure should be performed as early as possible to reduce the risk of venous thromboembolic events and sudden death. It is usually the recommended approach in patients with poor general condition, large vascular tumors, and anticipated extensive pelvic adhesions. One potential disadvantage of the two-stage procedure is that it may be associated with a higher rate of complications and also it may be more technically challenging secondary to a higher rate of scarring, particularly in the retrohepatic inferior vena cava. The two-stage procedure may be considered in individuals who have significant co-morbidities that preclude the patient from having a longer and more extensive procedure.

Discussants: Please provide details as to how to determine when one should consider cardiac bypass. What are the indications for thoracotomy?

Numerous strategies have been proposed to support the circulation with deep hypothermic circulatory arrest performed to allow safe removal of the tumor from the right atrium while minimizing the potential for major blood loss. One of the main factors impacting the surgical approach and strategy is the extent of thrombus extension and, in particular, its proximal level. Midline laparotomy combined with diaphragmotomy and pericardiotomy allows for clamping the intrapericardial portion of the inferior vena cava to perform thrombectomy from the inferior vena cava when performing extraction of the thrombus from the atrium. Large intra-atrial thrombi or extension into the right ventricle requires sternotomy and cardiopulmonary bypass.

Patients with tumor extension into the right atrium should be considered for cardiopulmonary bypass to decrease intra-operative blood loss and decrease the risk of air and tumor embolization. One should also note that tumor tissue in the suprarenal inferior vena cava has a higher propensity to being more adherent to the lumen, so additional precautions should be taken to ensure adequate exposure and proximal venous control when planning the tumor debulking procedure. Patients must be informed that cardiopulmonary bypass with moderate hypothermic arrest and cerebral perfusion may help decrease the likelihood of organ dysfunction, neurologic deficits, and severe coagulopathy that may occur with deep hypothermic arrest.

A thoracotomy may not be absolutely necessary if the masses can be pulled from the incision in the inferior vena cava. This is also more feasible when the tumor is not adherent to the vessel wall. It is strongly recommended to use transesophageal echocardiography rather than transthoracic echocardiography, since it can help the surgical team determine whether direct extraction of the entire tumor through the right atrium or inferior vena cava via a single incision is feasible, obviating the need for cardiopulmonary bypass, deep hypothermic arrest, or even sternotomy.

A decision to remove the tumor by thrombectomy was made at the multidisciplinary team discussion. Surgery was performed by total midline laparotomy in July 2012. This was done without opening the heart. The ascending colon was mobilized medially and a Kocher maneuver was performed. The ureters were mobilized and hysterectomy with bilateral adnexectomy was performed. A large tumor thrombus extending into the inferior vena cava was detected in the right ovarian vein. Sagittal diaphragmotomy and pericardiotomy were performed and inspection showed that the apex of the thrombus was in the right atrium. The Pringle maneuver was performed, and the renal veins and inferior vena cava below the renal veins were tourniqueted. The inferior vena cava was tourniqueted. Blood flow through the renal veins and in the inferior vena cava was stopped. The incision was performed on the anterior wall of the inferior vena cava directly below the level of the hepatic veins. The apex of the thrombus was extracted from the atrium 


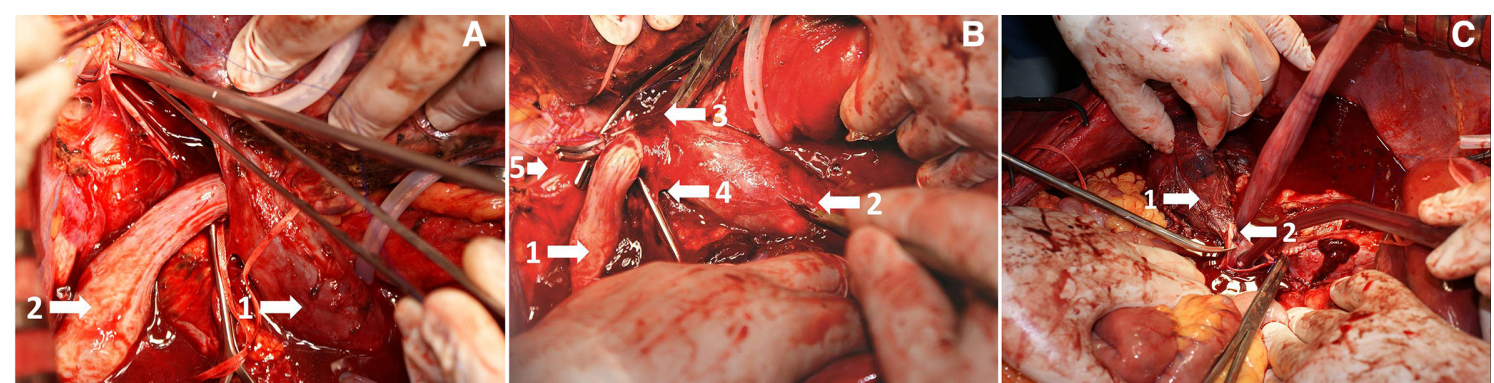

Figure 2 (A) Extraction of the intra-atrial component of the thrombus (1, vena cava; 2, tumor thrombus). (B) Dissection of the wall of the inferior vena cava at the site of thrombus fixation. Blood flow in the hepatic veins is restored (1, tumor thrombus; 2 , additional incision in the infrarenal section of the inferior vena cava; 3, double clamp on the inferior vena cava; 4, lumbar vein; 5 , right renal vein). (C) Final stage of thrombus removal from the resected wall of the inferior vena cava (1, right ovarian vein; 2 , right ovarian vein ostia).

and the thrombus was then removed from the inferior vena cava (Figure 2A).

The wall of the inferior vena cava was closed by continuous hand suture. The inferior vena cava was clamped below the suture line, after which blood flow through the renal veins and hepatoduodenal ligament was restored. The wall of the inferior vena cava was incised at the level of the right ovarian vein (Figure 2B), the ostium of the ovarian vein was resected and the thrombus with extracaval tumor masses was removed (Figure 2C). The wall of the inferior vena cava was sutured by continuous suture. Blood flow through the inferior vena cava was completely restored. The diaphragm was closed with interrupted sutures. The duration of surgery was $300 \mathrm{~min}$ and the blood loss was $1200 \mathrm{~mL}$. The patient stayed in the intensive care unit for 2 days. Total hospital stay was 9 days. There were no post-operative complications. Final pathology was consistent with intravenous leiomyomatosis. Given the positive hormone receptor status, we considered it reasonable to prescribe antiestrogen therapy with tamoxifen for 5 years and she discontinued this treatment in 2017. The patient's last visit was in September 2019 and there was no evidence of recurrence.

\section{Dr Andreeva}

Macropreparation consisted of the uterus with adnexa, tumor nodules, and thrombus, which was $24 \mathrm{~cm}$ long, with a diameter ranging from 1 to $3 \mathrm{~cm}$ (Figure 3). It is important to note that, in order to diagnose intravenous leiomyomatosis, morphologists

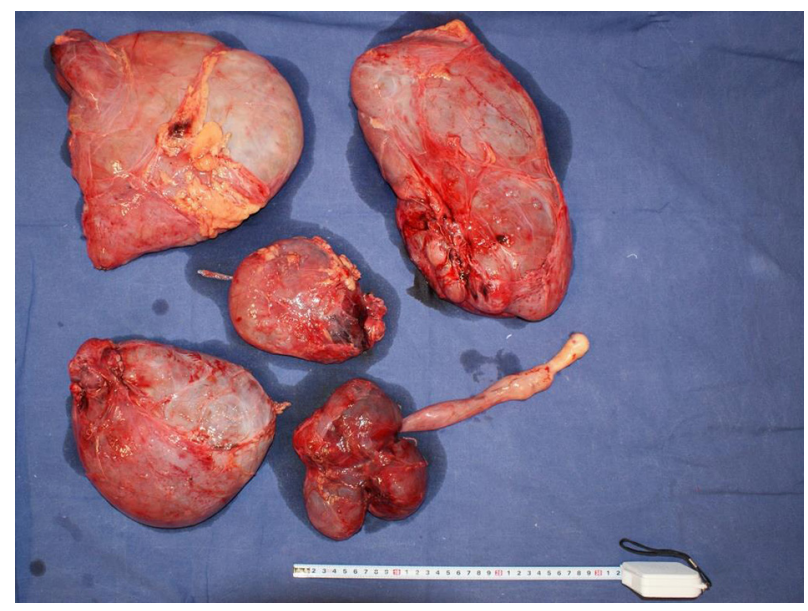

Figure 3 Intravenous leiomyomatosis: macropreparation. should identify tumor thrombi in the lumen of the veins using macroscopic analysis of the sample. The diagnosis was verified by microscopy analysis data. The microscopic image varied within the tumor: sections of typical leiomyoma alternated with fields of hyalinosis and foci of cellular structure. The wall of a large venous vessel contained a tumor thrombus which was shown to be a spindle cell tumor of a bundle structure, consisting of cells with elongated oval-shaped nuclei, areas of myxomatosis and hyalinosis of the stroma, apparent signs of cyst formation. The tumor cells formed strands extending through the lumina of the veins of large and small caliber and merging nodules (Figure 4A). Immunohistochemistry examination showed only expression of desmin, smooth muscle actin, caldesmon, Ki67-1\% (Figure 4B,C). Taking into account that morphologically this benign tumor often shows characteristics of a malignant neoplasm, we considered it feasible to perform molecular genetic testing. Fragment analysis performed using marker D10S218 showed the loss of heterozygosity (deletion of a lighter allele) in the tumor.

Discussants: What options were discussed with the patient regarding surveillance versus adjuvant treatment? What is the role of hormonal therapy as adjuvant treatment?

Post-operative surveillance is generally indicated every 3-6 months for the first 2 years after surgery, and then every 12 months thereafter. This is important as the rate of recurrence may be up to $30 \%{ }^{3}$ Although there is no consensus regarding which imaging modalities should be performed, it is generally recommended to perform a CT scan of the chest, abdomen, and pelvis at least once a year for the first 2 years of surveillance. Alternatively, an ultrasound of the peritoneal cavity, retroperitoneal space, pelvic cavity, including transvaginal and transanal examinations is recommended in the absence of CT scanning. Doppler ultrasound assessment of the inferior vena cava, iliac vessels, and veins of the lower limbs is suggested when there are symptoms suggestive of thromboembolic events.

The role of anti-estrogen therapy remains a topic of controversy in patients with intravenous leiomyomatosis. This is of particular importance for the use of hormonal treatment as preventive therapy for recurrence of disease; however, due to an insufficient number of publications to date, this issue has not been fully investigated and requires further consideration. 


\section{Case study}
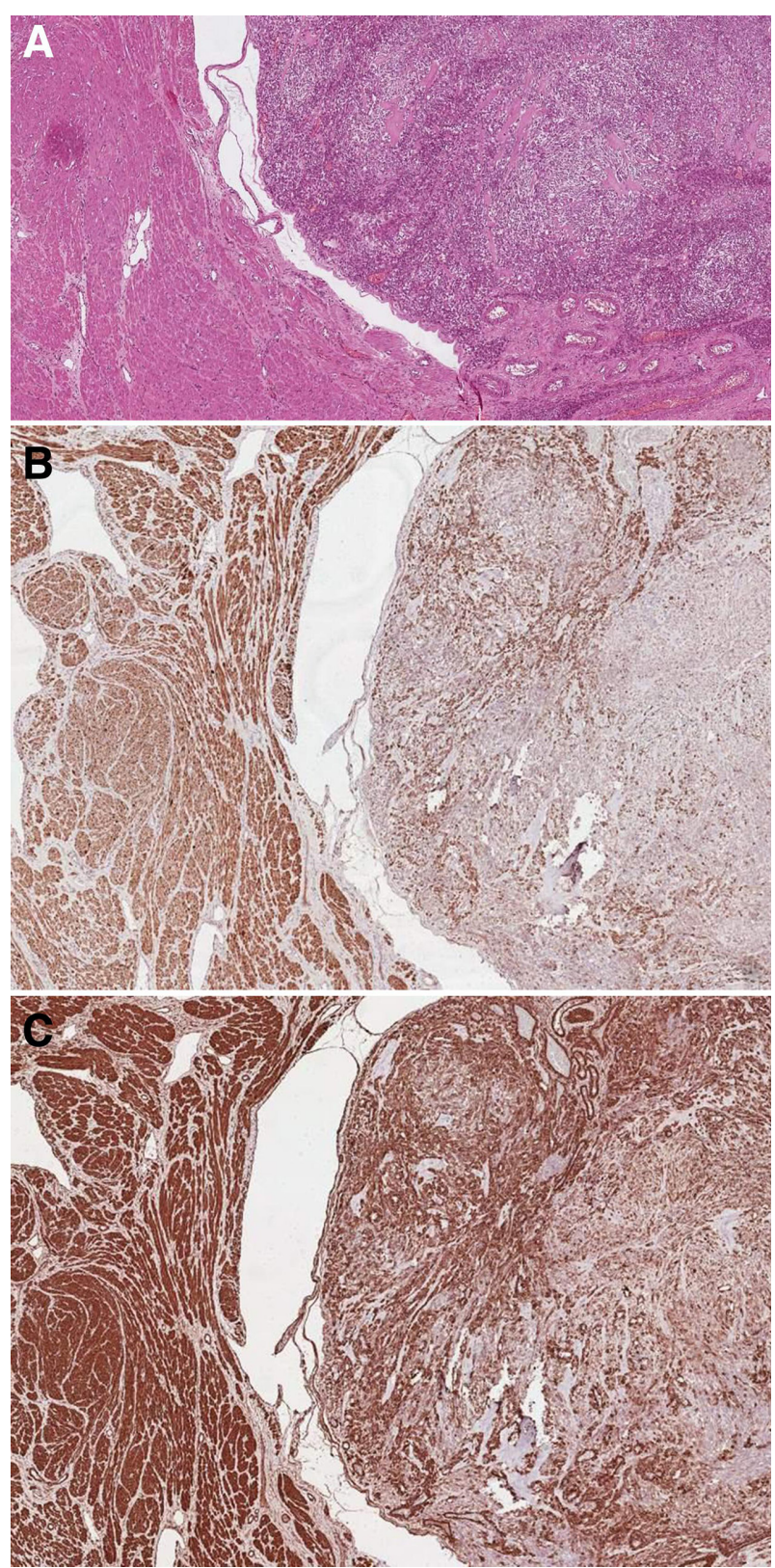

Figure 4 Intravenous leiomyomatosis. (A) Tumor in the lumen of the vein (hematoxylin and eosin staining, $\times 100)$. (B) Immunohistochemical reaction with antibodies to desmin (x100). (C) Immunohistochemical reaction with antibodies to smooth muscle actin $(\times 100)$.

\section{CLOSING SUMMARY}

Intravenous leiomyomatosis with intracardiac extension is a rare entity characterized by growth of a uterine neoplasm that extends through the uterine vascular channels, through the venous system, and ultimately into the cardiac chambers. The tumors may lead to significant physiologic disturbances and heart failure. In cases when the tumor thrombus reaches the pulmonary arteries, severe pulmonary embolism can occur. In some cases cardiac arrest may occur, particularly when the tumor involves the cardiac chambers. It commonly occurs in pre-menopausal women and approximately $83 \%$ have undergone a previous hysterectomy or myomectomy. ${ }^{2}$
It is more common in white women with antecedents of previous pregnancies and a history of hysterectomy or uterine leiomyoma.

Patients may present with abdominal pain, isolated syncopal episodes, pelvic mass, or a combination of these. Depending on the level and grade of occlusion of the inferior vena cava, the pathology can be asymptomatic or become an incidental finding in routine examinations, even in the group of patients with intracardiac intravenous leiomyomatosis. The most common pathway of extension for intravenous leiomyomatosis is unilateral through the iliac vein, although in some cases it may manifest with bilateral extension through the iliac and ovarian veins simultaneously. Intravenous leiomyomatosis is a benign neoplasm that is covered by endothelium which reduces its thrombogenic potential. It is thought that the turbulence within the vein does not allow it to adhere to the vessel wall. Tumor growth in the heart can increase the risk of sudden death attributable to the tricuspid valve orifice or right ventricular outflow tract obstruction.

The time from initial diagnosis of uterine leiomyoma to the index presentation of intravenous leiomyomatosis may vary, and some cases may present many years after initial diagnosis. The differential diagnosis of intravenous leiomyomatosis includes intravenous thrombus, Budd-Chiari syndrome, right atrial myxoma, renal cell carcinoma, primary leiomyosarcoma, or an endometrial stromal sarcoma. Almost $30 \%$ of patients are misdiagnosed on initial presentation as having right atrial myxoma. ${ }^{2}$ However, one should note that the overwhelming majority of atrial myxomas are on the left and intravenous leiomyomatosis is found on the right chambers. Treatment should be focused on removal of the primary site of disease, as well as the intracardiac burden and also on prevention of recurrence. This might include total hysterectomy, bilateral salpingo-oophorectomy, and resection of intravenous and intracardiac tumor.

The first description of the successful resection of intracardiac extension of a uterine leiomyoma was in 1974 by Mandelbaum and colleagues. ${ }^{4}$ However, the first complete removal of intravenous leiomyomatosis using a one-stage procedure was reported by Iverson et al in $1983^{5}$ Since then, several others have reported their experience with either the one-stage or twostage approach. In a large series reported by $\mathrm{Li}$ et $\mathrm{al},{ }^{2}$ the authors reported rates of complete removal in $54 \%$ of patients and a rate of intra-operative mortality of only $1.5 \%$. The ideal procedure is based on the individual patient and her presentation. It should be noted that formation of collateral circulation in the azygous system allows surgeons to ligate the infrarenal vena cava without compromising the venous return. It has been suggested that incomplete tumor resection is the most important determinant of disease recurrence.

Given that intravenous leiomyomatosis expresses high levels of estrogen and progesterone receptors, estrogen antagonists such as tamoxifen or letrozole may help prevent further tumor spread or recurrence. However, others have refuted this potential benefit. ${ }^{3}$ Additionally, there is no consensus as to what should be the standard recommendation for the duration of anti-estrogen therapy. Whether ovarian preservation is a risk factor for disease recurrence remains a topic of debate; however, given that intravenous leiomyomatosis is associated with high estrogen expression, intuitively one would consider that ovarian preservation is not recommended. 
Funding The authors have not declared a specific grant for this research from any funding agency in the public, commercial or not-for-profit sectors.

Competing interests None declared.

Patient consent for publication Not required.

Provenance and peer review Commissioned; externally peer reviewed.

\section{REFERENCES}

1 Altinok D, Yildiz YT, Tacal T, et al. MRI of intravascular leiomyomatosis extending to the heart. Eur Radiol 2000;10:871.
2 Li B, Chen X, Chu Y-D, et al. Intracardiac leiomyomatosis: a comprehensive analysis of 194 cases. Interact Cardiovasc Thorac Surg 2013;17:132-8.

3 Yu X, Zhang G, Lang J, et al. Factors associated with recurrence after surgical resection in women with intravenous leiomyomatosis. Obstet Gynecol 2016;128:1018-24.

4 Mandelbaum I, Pauletto FJ, Nasser WK. Resection of a leiomyoma of the inferior vena cava that produced tricuspid valvular obstruction. $J$ Thorac Cardiovasc Surg 1974;67:561-7.

5 Iverson LI, Lee J, Drew D, et al. Intravenous leiomyomatosis with cardiac extension. Tex Heart Inst J 1983;10:275-8. 